\title{
Coupling Systems for a New Type of Phase Synchronization
}

\author{
Florin D. Grosu, ${ }^{1}$ Adrian Bîrzu, ${ }^{2}$ Anca Lazar, ${ }^{3}$ and Ioan Grosu ${ }^{2,3}$ \\ ${ }^{1}$ Faculty of Medicine, Lucian Blaga University of Sibiu, Sibiu, Romania \\ ${ }^{2}$ Chemistry Department, Alexandru Ioan Cuza University, Iasi, Romania \\ ${ }^{3}$ Medical Bioengineering, Grigore T. Popa University of Medicine and Pharmacy, Iasi, Romania
}

Correspondence should be addressed to Ioan Grosu; ioan.grosu@chem.uaic.ro

Received 12 May 2016; Revised 17 July 2016; Accepted 9 August 2016

Academic Editor: Jonathan N. Blakely

Copyright (C) 2016 Florin D. Grosu et al. This is an open access article distributed under the Creative Commons Attribution License, which permits unrestricted use, distribution, and reproduction in any medium, provided the original work is properly cited.

Using the usual phase in plane, we propose a general method to design coupling between systems that will exhibit phase synchronization. Numerical results are shown for Lorenz systems. Phase synchronization and antiphase synchronization are equally probable depending on initial conditions. A new network with Lorenz phase synchronized system is obtained.

\section{Introduction}

Nonlinear dynamics brought to light the rich dynamics of nonlinear systems including chaotic behavior. By coupling two nonlinear systems a new nonlinear system with an increased dimension and richer dynamics is obtained. The couplings can be suggested by reality (like diffusive coupling) or can result from the design in order to achieve a certain task. Depending on the coupling at least two distinctive cases can occur after the coupling is on: synchronization $[1,2]$ and Amplitude Death (AD) [3, 4]. Synchronization is a fundamental phenomenon observed in nature and science and used in engineering and medicine $[1,2]$. There are several types of synchronization (complete synchronization, lag synchronization, projective synchronization, and generalized synchronization including amplification/attenuation). We mention here just some results that show how to design the couplings for synchronization [5-13]. A weaker type of synchronization is phase synchronization (PS) as locking of the phase of the coupled systems, while their amplitudes remain uncorrelated. The great interest for this kind of synchronization has been triggered by the seminal paper [14]. For chaotic systems it is difficult to introduce a phase. In [14], the phase was introduced by using the analytic signal concept. Many results on PS are observed for chaotic attractors with rather coherent phase dynamics $[1,2]$. Phase coherent dynamics is a strong limitation for applications. A step forward is the study of the effect of noncoherence on the onset PS [15] by using a phase based on the general idea of curvature of an arbitrary curve.
Also PS was obtained by using active control and by nonlinear state observer algorithm [16, 17] when the difference of two homologous variables is constant. However, a concern has arisen recently related to the use of the analytic signal concept [18].

In this paper we adopt a new strategy. By using the usual definition of phase in plane we deduce a system that governs the dynamics of the phase differences. From this dynamics we search for coupling terms that assure that the phase differences become asymptotically zero. The method is general but it does not assure that we can find coupling terms for any systems. In addition, it is not assured that the dynamics of the coupled system is bounded. For Lorenz system, the method gives good results. Coupling for two Lorenz systems suggests an all-to-all coupling for a network of PS and antiphase synchronized (APS) systems. Such a network can have different outputs depending on initial conditions.

The organization of this paper is as follows. In Section 2, the general theory is presented, where the system that governs the dynamics of the phase differences is deduced. Section 3 gives the results of the theory applied to Lorenz systems with numerical results. Section 4 contains several comments. Finally, conclusions are presented in Section 5.

\section{Theory}

Without losing generality, we consider 3 dimensional systems $\mathrm{d} s / \mathrm{d} t=F(s)$ with $s=(x, y, z)^{\mathrm{T}}$ like Lorenz, Chen, Rössler, 
Sprott, and so forth. $F(s)=L s+N(s)$, where $L$ is a $3 \times 3$ constant matrix and $N(s)$ is a $3 \times 1$ vector which contains nonlinear terms. Two systems that can be coupled by terms $u_{1}$ and $u_{2}$ have equations:

$$
\begin{aligned}
& \frac{\mathrm{d} s_{1}}{\mathrm{~d} t}=L s_{1}+N\left(s_{1}\right)+u_{1}\left(s_{1}, s_{2}\right) \\
& \frac{\mathrm{d} s_{2}}{\mathrm{~d} t}=L s_{2}+N\left(s_{2}\right)+u_{2}\left(s_{1}, s_{2}\right) .
\end{aligned}
$$

We need to determine $u_{1}$ and $u_{2}$ in such a manner that the two systems will reach PS in at least two pairs of variables (let us say $\left.x_{i}, y_{i}, i=1,2\right)$. As phase we adopt the usual phase in plane $O x_{i} y_{i}: \theta_{i}=\tan ^{-1}\left(y_{i} / x_{i}\right), i=1,2$. The cross product $P=s_{1} \times s_{2}$ has the following components:

$$
\left(P_{1}, P_{2}, P_{3}\right)=\left(z_{2} y_{1}-z_{1} y_{2}, x_{2} z_{1}-x_{1} z_{2}, x_{1} y_{2}-x_{2} y_{1}\right) \text {. }
$$

The component $P_{3}$ depends on phase difference:

$$
P_{3}=\left(x_{1}^{2}+y_{1}^{2}\right)^{1 / 2}\left(x_{2}^{2}+y_{2}^{2}\right)^{1 / 2} \sin \left(\theta_{2}-\theta_{1}\right) \text {. }
$$

$P_{3}=0$ means $x_{1}^{2}+y_{1}^{2}=0$ (Amplitude Death) or $x_{2}^{2}+y_{2}^{2}=0$ (Amplitude Death) or $\sin \left(\theta_{2}-\theta_{1}\right)=0 \Rightarrow \theta_{2}=\theta_{1}$; that is, PS or $\theta_{2}=\theta_{1}+\pi$, that is, APS.

The dynamics of $P_{i}$ can be obtained by using the original dynamics (1a) and (1b) and the components $P_{i}$ given by (2):

$$
\begin{aligned}
& \frac{\mathrm{d} P_{1}}{\mathrm{~d} t}=\left(L_{22}+L_{33}\right) P_{1}-L_{21} P_{2}-L_{31} P_{3}+M_{1}, \\
& \frac{\mathrm{d} P_{2}}{\mathrm{~d} t}=-L_{12} P_{1}+\left(L_{11}+L_{33}\right) P_{2}-L_{32} P_{3}+M_{2}, \\
& \frac{\mathrm{d} P_{3}}{\mathrm{~d} t}=-L_{13} P_{1}-L_{23} P_{2}+\left(L_{11}+L_{22}\right) P_{3}+M_{3},
\end{aligned}
$$

or

$$
\frac{\mathrm{d} P^{\mathrm{T}}}{\mathrm{d} t}=L^{*} P^{\mathrm{T}}+M,
$$

where $M=\left(M_{1}, M_{2}, M_{3}\right)^{\mathrm{T}}$ with $M$ containing all nonlinear terms, $N(s)$, and coupling terms of $u_{1}$ and $u_{2}$.

Now we can give a partial answer to the question [15] "It is still unknown which type of chaotic oscillators can be synchronized in phase"? The general and partial answer is that system (4a), system (4b), and system (4c) or system (5) contains information on the PS (with phase $\theta_{i}=$ $\left.\tan ^{-1}\left(y_{i} / x_{i}\right), i=1,2\right)$. This is the main result of this research and is general because (4a), (4b), and (4c) are general. The method of finding $u_{1}$ and $u_{2}$ depends on the system and can be more or less complicated (see Section 4).

If we find coupling terms $u_{1}$ and $u_{2}$ in such a manner that $M_{i}=0$ and $\mathrm{d} P_{i} / \mathrm{d} t=-k P_{i}(k>0)$, then we have PS or AD as above. If one line in $L^{*}$ (in (5)) contains just zeros, then we can add and subtract $-k P_{i}$ and look for coupling terms to have $k P_{i}+M_{i}=0$.

If by choosing $u_{1}$ and $u_{2}$ we can have $M_{i}=M_{j}=0$ and the system is $\mathrm{d} P_{i} / \mathrm{d} t=a_{1} P_{i}+a_{2} P_{j}$ and $\mathrm{d} P_{j} / \mathrm{d} t=a_{3} P_{i}+a_{4} P_{j}$ and if $a_{i}, i=1-4$, assures that $P_{i} \rightarrow 0$ and $P_{j} \rightarrow 0$, then all variables are PS (or APS).

If we find $u_{1}$ and $u_{2}$, where all $M_{i}=0, i=1,2,3$, and $L^{*}$ (in (5)) is a Hurwitz matrix (a matrix with negative real part eigenvalues), then $P_{i} \rightarrow 0, i=1,2,3$, and all variables will be PS and amplitudes are uncorrelated.

At the eye inspection of the temporal pattern of two systems PS or APS (let us say $x_{1}$ and $x_{2}$ and $y_{1}$ and $y_{2}$ ) we will observe (see Figures 1 and 2; numerical results) that the homologous variables cross the time axis at the same time as expected from (2). This conclusion can have practical importance at the investigation of real data.

To check numerically PS we can use coefficients $\left(c_{1}, c_{2}\right.$, and $c_{3}$ ) with

$$
c_{3}=\frac{x_{1} x_{2}+y_{1} y_{2}}{\left(x_{1}^{2}+y_{1}^{2}\right)^{1 / 2}\left(x_{2}^{2}+y_{2}^{2}\right)^{1 / 2}}
$$

and similar expressions for $c_{1}$ and $c_{2}$. For $c_{3}$, we have

$$
c_{3}=\cos \left(\theta_{2}-\theta_{1}\right)
$$

For PS, $c_{3}=1$ and $c_{3}=-1$ means APS. Again, this result can be of practical use. There can be identified two limitations of this general strategy. Firstly, the coupling terms do not assure that the dynamics of the coupled systems are bounded. Secondly, the coupling terms are not unique, but this can be an advantage when the first limitation occurs.

\section{Phase Synchronized Lorenz Systems}

We apply the above strategy to Lorenz systems $(\mathrm{d} x / \mathrm{d} t=$ $-\sigma x+\sigma y, \mathrm{~d} y / \mathrm{d} t=r x-y-x z$, and $\mathrm{d} z / \mathrm{d} t=-b z+x y)$. In this case,

$$
\begin{aligned}
& L=((-\sigma, \sigma, 0),(r,-1,0),(0,0-b))^{\mathrm{T}}, \\
& L^{*}=((-b-1,-r, 0),(\sigma,-\sigma-b, 0),(0,0,-\sigma-1))^{\mathrm{T}}, \\
& N(s)=(0,-x z, x y)^{\mathrm{T}} .
\end{aligned}
$$

Equation (4c) with (9) gives

$$
\frac{\mathrm{d} P_{3}}{\mathrm{~d} t}=-(\sigma+1) P_{3}+M_{3}
$$

with

$$
\begin{aligned}
M_{3}= & y_{2} u_{11}-y_{1} u_{21}+x_{1} u_{22}-x_{2} u_{12} \\
& +x_{1} x_{2}\left(z_{1}-z_{2}\right) .
\end{aligned}
$$

We need to find coupling terms to have $M_{3}=0$. 

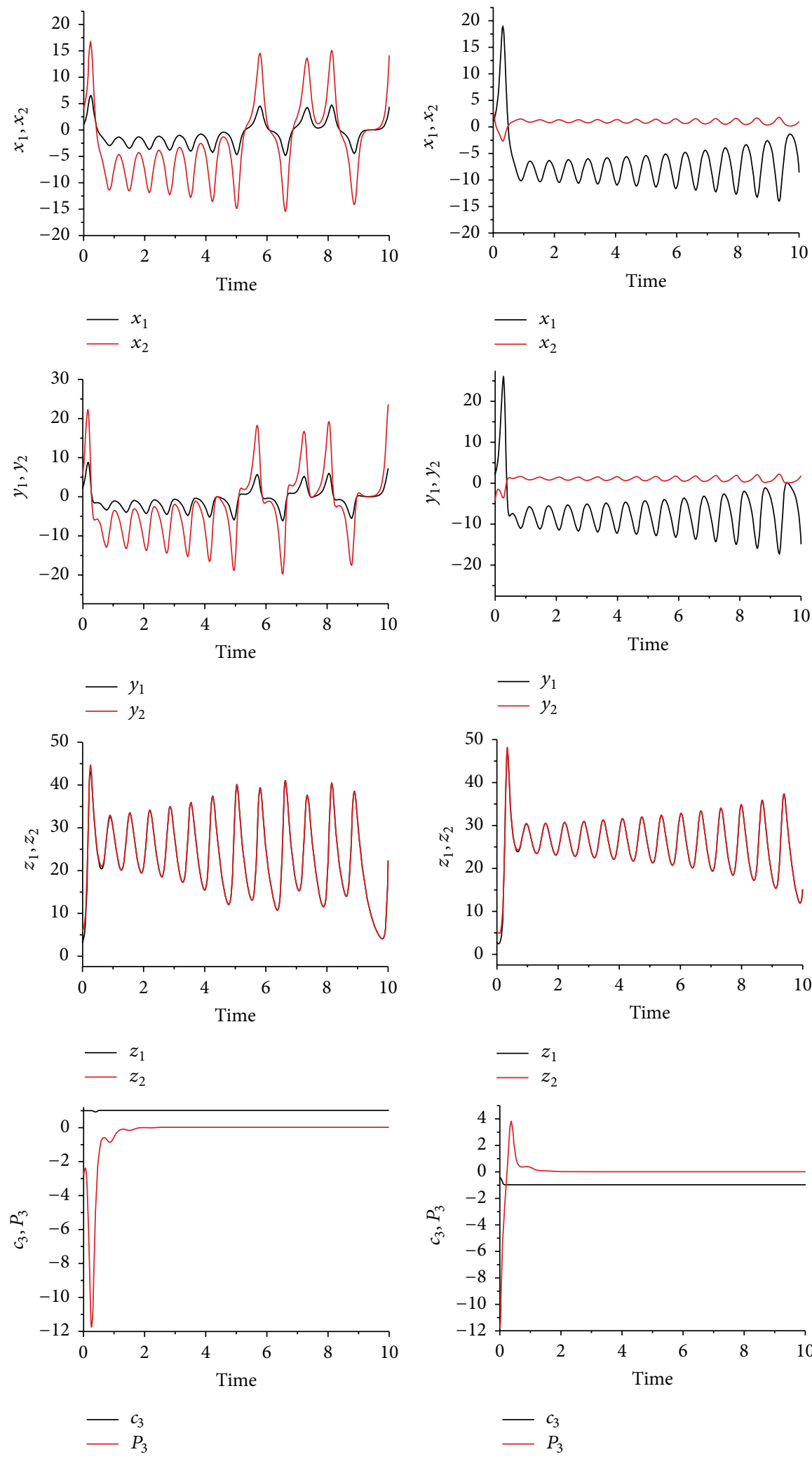

(a)

(b)

Figure 1: Lorenz systems coupled in Case 1 (see text; $\mathrm{d} x_{1} / \mathrm{d} t=-\sigma x_{1}+\sigma y_{1}, \mathrm{~d} y_{1} / \mathrm{d} t=r x_{1}-y_{1}-x_{1} z_{1}, \mathrm{~d} z_{1} / \mathrm{d} t=-b z_{1}+x_{1} y_{1}+x_{2} y_{2}$, $\mathrm{d} x_{2} / \mathrm{d} t=-\sigma x_{2}+\sigma y_{2}, \mathrm{~d} y_{2} / \mathrm{d} t=r x_{2}-y_{2}-x_{2} z_{2}$, and $\left.\mathrm{d} z_{2} / \mathrm{d} t=-b z_{2}+x_{1} y_{1}+x_{2} y_{2}\right)$. Initial conditions. (a) $\left(x_{1}, y_{1}, z_{1}, x_{2}, y_{2}, z_{2}\right)(0)=(1,2,3,4,5,6)$, $c_{3}=1$, and $P_{3}=0$, PS. (b) $\left(x_{1}, y_{1}, z_{1}, x_{2}, y_{2}, z_{2}\right)(0)=(1,2,3,4,-5,6), c_{3}=-1$, and $P_{3}=0$, APS. In both cases, $x_{1}$ and $x_{2}$ (resp., $y_{1}$ and $\left.y_{2}\right)$ cross the time axis at the same moment. $z_{1}=z_{2}$ asymptotically. 

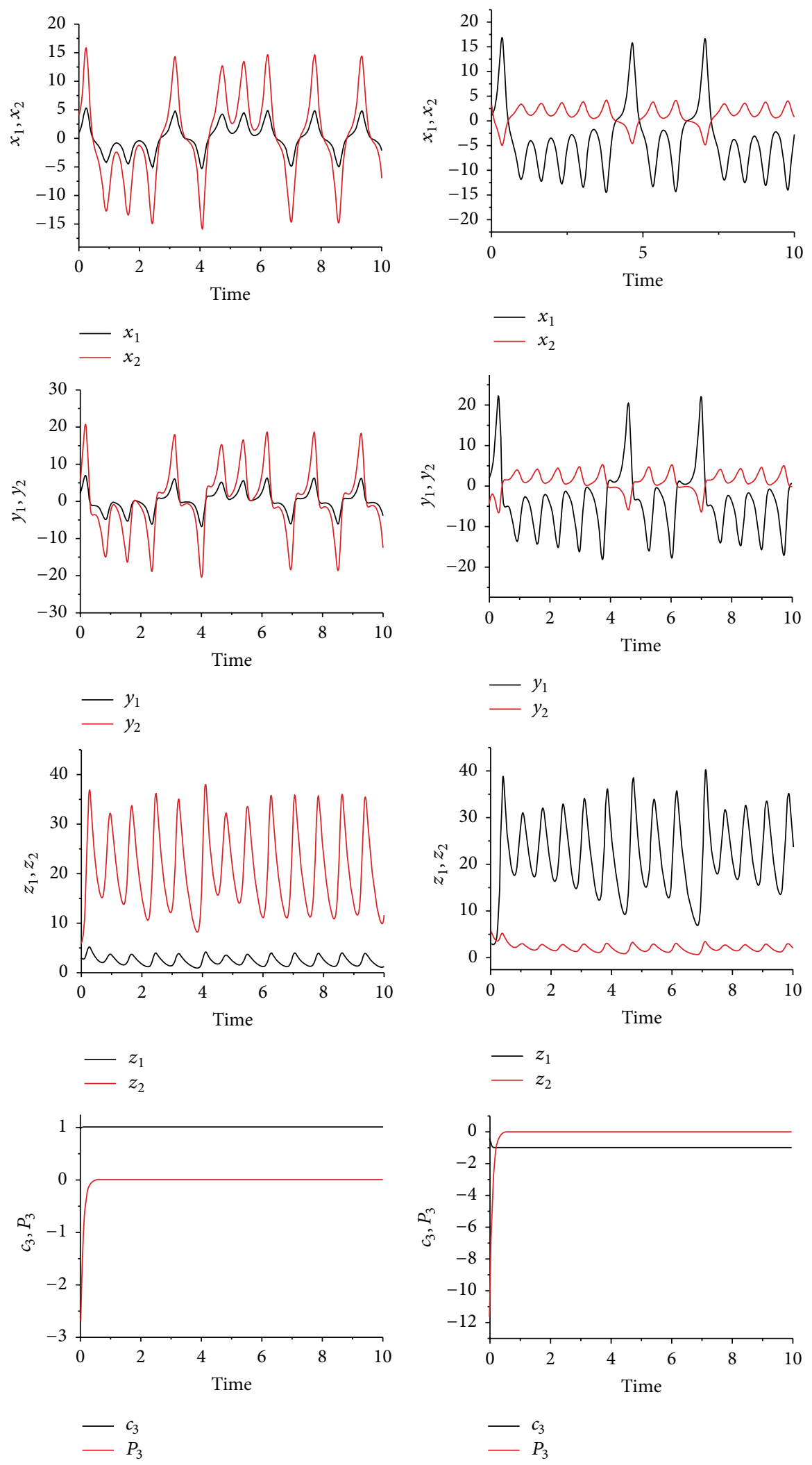

(a)

(b)

Figure 2: Lorenz systems coupled in Case $2\left(\mathrm{~d} x_{1} / \mathrm{d} t=-\sigma x_{1}+\sigma y_{1}, \mathrm{~d} y_{1} / \mathrm{d} t=r x_{1}-y_{1}-x_{1} z_{1}-x_{1} z_{2}, \mathrm{~d} z_{1} / \mathrm{d} t=-b z_{1}+x_{1} y_{1}, \mathrm{~d} x_{2} / \mathrm{d} t=-\sigma x_{2}+\sigma y_{2}\right.$, $\mathrm{d} y_{2} / \mathrm{d} t=r x_{2}-y_{2}-x_{2} z_{2}-x_{2} z_{1}$, and $\left.\mathrm{d} z_{2} / \mathrm{d} t=-b z_{2}+x_{2} y_{2}\right)$. Initial conditions. (a) $\left(x_{1}, y_{1}, z_{1}, x_{2}, y_{2}, z_{2}\right)(0)=(1,2,3,4,5,6), c_{3}=1$, and $P_{3}=0$, PS. (b) $\left(x_{1}, y_{1}, z_{1}, x_{2}, y_{2}, z_{2}\right)(0)=(1,2,3,4,-5,6), c_{3}=-1$, and $P_{3}=0$, APS. In both cases $z_{i}, i=1,2$, are uncorrelated; similar variables cross the time axis at the same moment. 
Three cases can be identified.

Case 1. One has

$$
\begin{aligned}
& u_{11}=u_{12}=u_{21}=u_{22}=0, \\
& u_{13}=x_{2} y_{2}, \\
& u_{23}=x_{1} y_{1} .
\end{aligned}
$$

In this case, subtracting the 3rd equations of (1a) and (1b), we have $\mathrm{d}\left(z_{2}-z_{1}\right) / \mathrm{d} t=-b\left(z_{2}-z_{1}\right)$ so $z_{2} \rightarrow z_{1}$ and, from (12), $M_{3}=0$ and, from (11), $P_{3} \rightarrow 0$. The final state will be PS or APS depending on initial conditions like in Figure 1. Numerical results are given in Figure 1 with $(\sigma, r, b)=(10,28,8 / 3)$. Two examples are given: one PS and one APS from different initial conditions. $P_{3}=0$ means PS or APS; $c_{3}=1$ means PS and $c_{3}=-1$ means APS.

Case 2. One has

$$
\begin{aligned}
& u_{11}=u_{21}=0, \\
& u_{22}=-x_{2} z_{1}, \\
& u_{12}=-x_{1} z_{2}, \\
& u_{13}=u_{23}=0 .
\end{aligned}
$$

Numerical results are given in Figure 2.

Case 3. One has

$$
\begin{aligned}
& u_{11}=y_{1}, \\
& u_{21}=y_{2}, \\
& u_{12}=-x_{1} z_{2}, \\
& u_{22}=-x_{2} z_{1}, \\
& u_{13}=u_{23}=0 .
\end{aligned}
$$

Numerical results (not shown) are qualitatively similar to Case 2.

\section{Discussions}

In our numerical experiments, we did not find any $\mathrm{AD}$ and we do not have any indications how or if it can be found. In some cases one oscillation is much smaller than the other but not AD. This is worthy to be deeply investigated.

For Chen systems, the results are very similar to Lorenz system.

Unfortunately, we did not find reasonable coupling terms for Rössler system using a direct method like we used for Lorenz system. Other methods should be tried. Here we need to clarify this problem. We report here that we did not find coupling terms for Rössler by simple eye inspection as we obtained for Lorenz system. In fact, in general, we need to find coupling terms in (4a), (4b), and (4c) or (5) which assures that components of $P$ go to zero. As an ultimate method the OPCL (open-plus-closed-loop) method [19] can be used which can offer driving for any system to reach any desired dynamics. The coupling terms can be complicated but for engineering applications it is important that they can be possible. This will be elaborated (with examples) elsewhere.

For system $A$ from Sprott collection $[20](\mathrm{d} x / \mathrm{d} t=y$, $\mathrm{d} y / \mathrm{d} t=-x+y z$, and $\left.\mathrm{d} z / \mathrm{d} t=1-y^{2}\right), L=((0,1,0)$, $(-1,0,0),(0,0,0))^{\mathrm{T}}, N(s)=\left(0, y z, 1-y^{2}\right)^{\mathrm{T}}$, and $L^{*}=$ $((0,1,0),(-1,0,0),(0,0,0))^{\mathrm{T}}$. Equation $(4 \mathrm{c})$ in this case is $\mathrm{d} P_{3} / \mathrm{d} t=M_{3} ; M_{3}=y_{2} z_{2} x_{1}+u_{22} x_{1}+y_{2} u_{11}-u_{21} y_{1}-x_{2} y_{1} z_{1}-$ $x_{2} u_{12}$. So we subtract and add $k P_{3}$ and find the coupling terms from $k P_{3}+M_{3}=0$ as

$$
\begin{aligned}
& u_{11}=-z_{2} x_{1}, \\
& u_{21}=-x_{2} z_{1}, \\
& u_{12}=-k y_{1}, \\
& u_{22}=-k y_{2}, \\
& u_{13}=u_{23}=0 .
\end{aligned}
$$

Numerical results (not shown) for initial condition

$$
\left(x_{1}, y_{1}, z_{1}, x_{2}, y_{2}, z_{2}\right)(0)=(1,2,3,2,-3,4)
$$

give bounded dynamics just for $k>2.1$.

Initial conditions in connection with PS and APS were considered also in [21]. Antiphase synchronization (for diffusive coupling) was investigated in [22].

Case 1 for Lorenz systems (13) suggests a network of PS and APS systems given by

$$
\begin{aligned}
& \frac{\mathrm{d} x_{i}}{\mathrm{~d} t}=-\sigma x_{i}+\sigma y_{i}, \\
& \frac{\mathrm{d} y_{i}}{\mathrm{~d} t}=r x_{i}-y_{i}-x_{i} z_{i}, \\
& \frac{\mathrm{d} z_{i}}{\mathrm{~d} t}=-b z_{i}+\sum_{j=1}^{N} x_{j} y_{j} .
\end{aligned}
$$

The coupling here is a nonlinear global coupling used recently [23]. Here $N$ is the number of systems coupled allto-all. In this case all systems of the network organize in 2 PS clusters and APS between them. Such a network can have a very interesting output. If the output is $X=\sum_{j=1}^{N} x_{j}$, $Y=\sum_{j=1}^{N} y_{j}$, and $Z=\sum_{j=1}^{N} z_{j}$, then the output will depend on the initial conditions. If all systems are PS, then the output will be maximum, and if the two PS clusters (that are APS one to another) are with nearly equal numbers of systems, then the output will be very small in comparison to the previous case. Figure 3 gives numerical results for $N=3$ Lorenz systems starting from different initial conditions. For similar initial conditions the output is very different. As a common characteristic, all variables cross the time axis at the same moment.

This property of crossing time axis at the same moment can be searched by eye inspection in real signal (like EKG, 

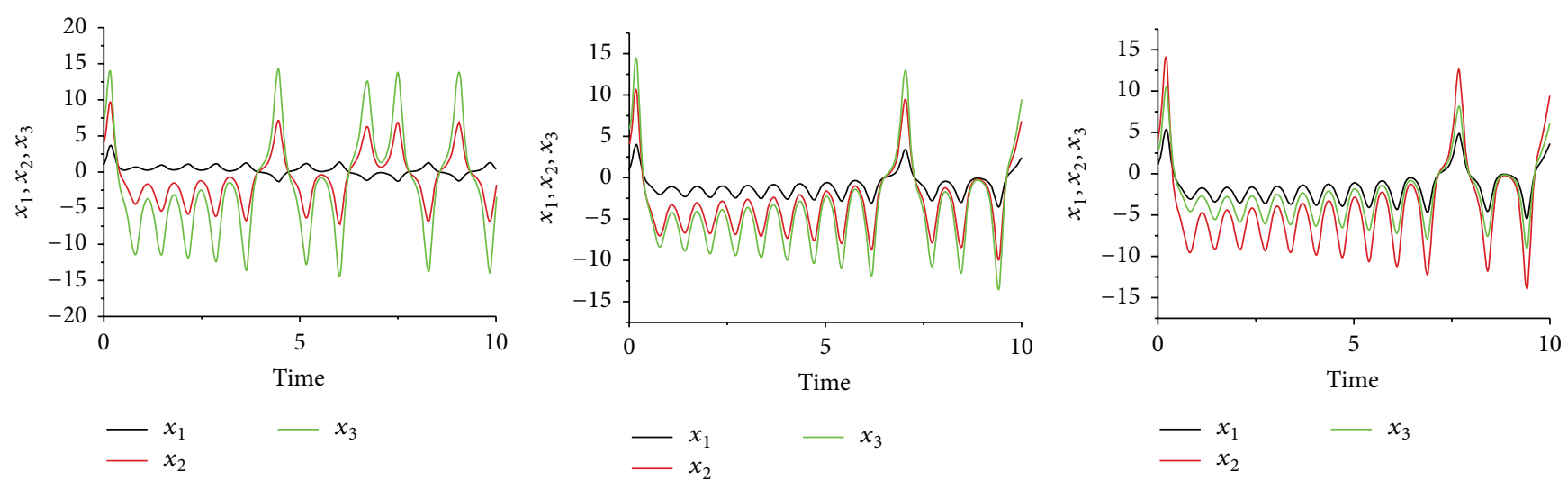

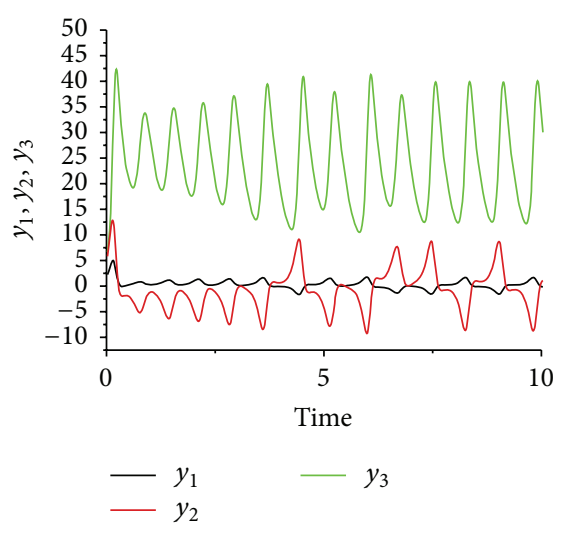

(a)

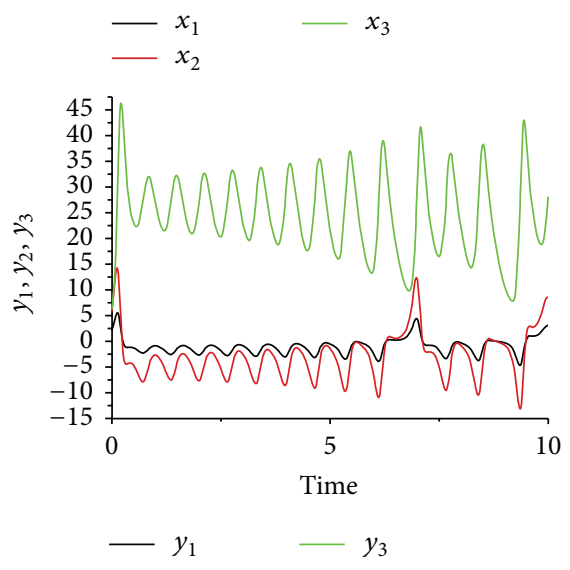

(b)
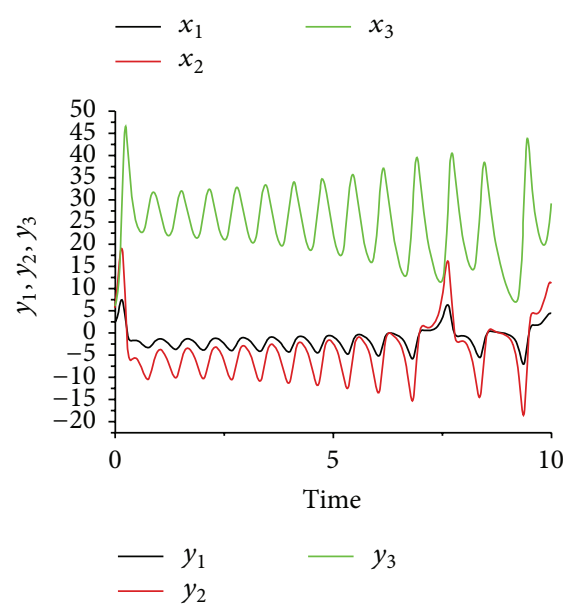

(c)

FIGURE 3: Three Lorenz systems coupled as in (18) for different initial conditions. (a) $\left(x_{1}, y_{1}, z_{1}, x_{2}, y_{2}, z_{2}, x_{3}, y_{3}, z_{3}\right)(0)=(1,2,3,4,5,6,7,8,9)$, 2 clusters (Systems 2 and 3 are PS (1st cluster) in antiphase with system 1 (2nd cluster)); (b) $\left(x_{1}, y_{1}, z_{1}, x_{2}, y_{2}, z_{2}, x_{3}, y_{3}, z_{3}\right)(0)=$ $(1,2,3,4,5,6,8,5,4)$, all PS. (c) $\left(x_{1}, y_{1}, z_{1}, x_{2}, y_{2}, z_{2}, x_{3}, y_{3}, z_{3}\right)(0)=(1,2,3,4,5,6,3,2,1)$, all PS.

EEG, EMG, or others) and if it happens then a model with this type of coupling can be used.

Based on this general strategy other results will be presented elsewhere.

\section{Conclusions}

In this paper new strategy for phase synchronization is used a. Phase is not calculated effectively but a coupling is searched which assures that the two current phases are equal. Phase is the usual definition in plane: $\left(\theta_{i}=\tan ^{-1}\left(y_{i} / x_{i}\right), i=1,2\right)$. The main result with possible practical importance is that the PS or APS signals pass zero value at the same moment at an eye inspection. The present phase synchronized Lorenz systems can be used as a tool for signal processing of real data as complete synchronized Lorenz systems have been used recently [24]. Deviation from $c_{3}=1$ measures the difference between two signals; results can be much better because $c_{3}$ is adimensional.

\section{Competing Interests}

The authors declare that they have no competing interests.

\section{References}

[1] A. Pikovsky, M. Rosenblum, and J. Kurths, Synchronization: A Universal Concept in Nonlinear Sciences, Cambridge University Press, New York, NY, USA, 2003.

[2] S. Boccaletti, J. Kurths, G. Osipov, D. L. Valladares, and C. S. Zhou, "The synchronization of chaotic systems," Physics Reports, vol. 366, no. 1-2, pp. 1-101, 2002.

[3] G. Saxena, A. Prasad, and R. Ramaswamy, "Amplitude death: the emergence of stationarity in coupled nonlinear systems," Physics Reports, vol. 521, no. 5, pp. 205-228, 2012.

[4] A. Koseska, E. Volkov, and J. Kurths, "Oscillation quenching mechanisms: amplitude vs. oscillation death," Physics Reports, vol. 531, no. 4, pp. 173-199, 2013.

[5] I. Grosu, "Robust synchronization," Physical Review E, vol. 56, no. 3, pp. 3709-3712, 1997.

[6] A. I. Lerescu, N. Constandache, S. Oancea, and I. Grosu, "Collection of master-slave synchronized chaotic systems," Chaos, Solitons and Fractals, vol. 22, no. 3, pp. 599-604, 2004.

[7] I. Grosu, E. Padmanaban, P. K. Roy, and S. K. Dana, "Designing coupling for synchronization and amplification of chaos," Physical Review Letters, vol. 100, no. 23, Article ID 234102, 2008.

[8] I. Grosu, R. Banerjee, P. K. Roy, and S. K. Dana, "Design of coupling for synchronization of chaotic oscillators," Physical 
Review E: Statistical, Nonlinear, and Soft Matter Physics, vol. 80, no. 1, Article ID 016212, 2009.

[9] P. K. Roy, C. Hens, I. Grosu, and S. K. Dana, "Engineering generalized synchronization in chaotic oscillators," Chaos, vol. 21, no. 1, Article ID 013106, 2011.

[10] D. Ghosh, I. Grosu, and S. K. Dana, "Design of coupling for synchronization in time-delayed systems," Chaos, vol. 22, no. 3, Article ID 033111, 8 pages, 2012.

[11] S. Oancea, F. Grosu, A. Lazar, and I. Grosu, "Master-slave synchronization of Lorenz systems using a single controller," Chaos, Solitons and Fractals, vol. 41, no. 5, pp. 2575-2580, 2009.

[12] H. Liu, Z. Zhu, H. Yu, and Q. Zhu, "Modified projective synchronization between different fractional-order systems based on open-plus-closed-loop control and its application in image encryption," Mathematical Problems in Engineering, vol. 2014, Article ID 567898, 10 pages, 2014.

[13] Q. Han, H. Zhang, and J. Liu, "Nonlinear dynamics of controlled synchronizations of manipulator system," Mathematical Problems in Engineering, vol. 2014, Article ID 691464, 9 pages, 2014.

[14] M. G. Rosenblum, A. S. Pikovsky, and J. Kurths, "Phase synchronization of chaotic oscillators," Physical Review Letters, vol. 76, no. 11, pp. 1804-1807, 1996.

[15] G. V. Osipov, B. Hu, C. Zhou, M. V. Ivanchenko, and J. Kurths, "Three types of transitions to phase synchronization in coupled chaotic oscillators," Physical Review Letters, vol. 91, no. 2, Article ID 024101, 2003.

[16] M.-C. Ho, Y.-C. Hung, and C.-H. Chou, "Phase and anti-phase synchronization of two chaotic systems by using active control," Physics Letters A, vol. 296, no. 1, pp. 43-48, 2002.

[17] J. Meng and X.-Y. Wang, "Nonlinear observer based phase synchronization of chaotic systems," Physics Letters A, vol. 369, no. 4, pp. 294-298, 2007.

[18] M.-C. Lu, H.-C. Ho, C.-A. Chan, C.-J. Liu, J.-S. Lih, and M.C. Ho, "Phase synchronization is the amplified result by the Hilbert transform," Mathematical Problems in Engineering, vol. 2015, Article ID 640107, 3 pages, 2015.

[19] E. A. Jackson and I. Grosu, "An open-plus-closed-loop (OPCL) control of complex dynamic systems," Physica D. Nonlinear Phenomena, vol. 85, no. 1-2, pp. 1-9, 1995.

[20] J. C. Sprott, "Some simple chaotic flows," Physical Review E, vol. 50, no. 2, pp. R647-R650, 1994.

[21] Y. Wu, N. Wang, L. Li, and J. Xiao, "Anti-phase synchronization of two coupled mechanical metronomes," Chaos, vol. 22, no. 2, Article ID 023146, 2012.

[22] W. Liu, J. Xiao, X. Qian, and J. Yang, "Antiphase synchronization in coupled chaotic oscillators," Physical Review E, vol. 73, no. 5, Article ID 057203, 2006.

[23] L. Schmidt and K. Krischer, "Clustering as a prerequisite for chimera states in globally coupled systems," Physical Review Letters, vol. 114, no. 3, Article ID 034101, 2015.

[24] R. Ghaffari, I. Grosu, D. Iliescu, E. Hines, and M. Leeson, "Dimensionality reduction for sensory datasets based on master-slave synchronization of Lorenz system," International Journal of Bifurcation and Chaos, vol. 23, no. 5, Article ID 1330013, 2013. 


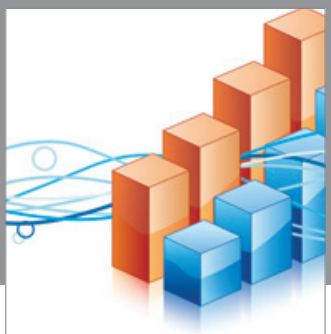

Advances in

Operations Research

vatem alat4

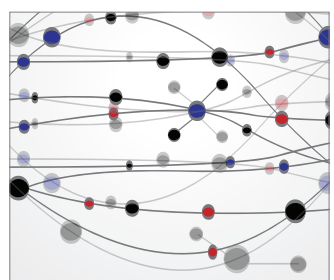

\section{The Scientific} World Journal
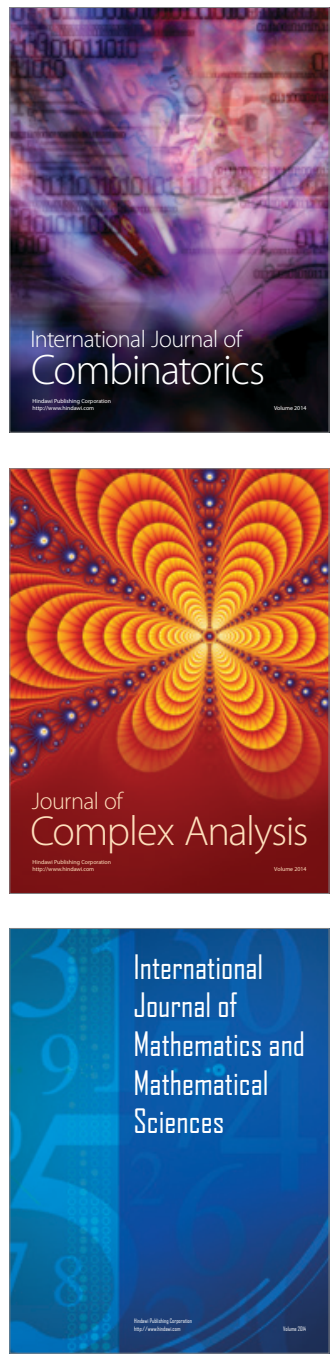
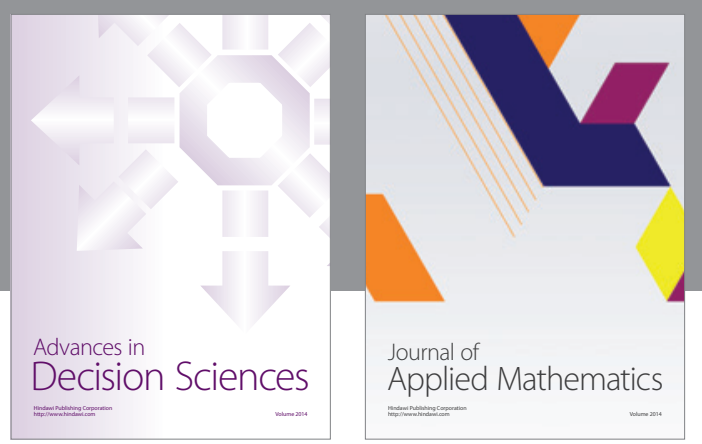

Algebra

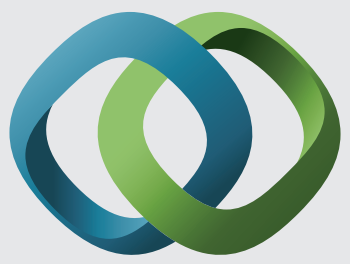

\section{Hindawi}

Submit your manuscripts at

http://www.hindawi.com
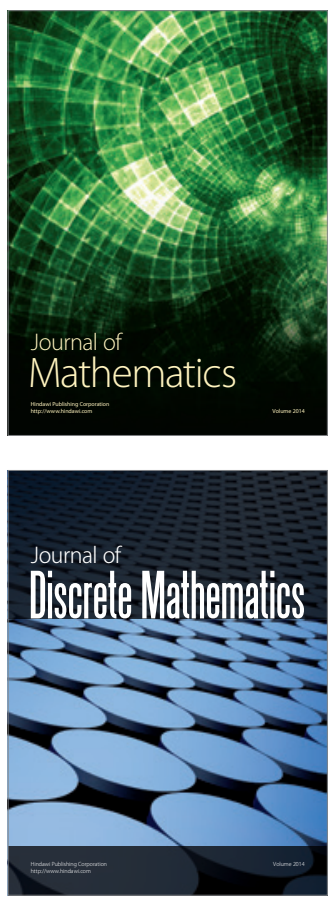

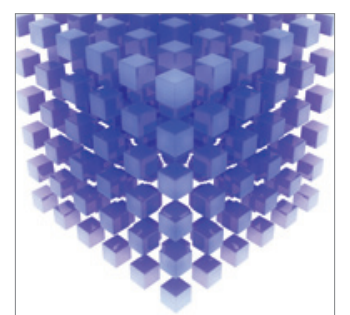

Mathematical Problems in Engineering
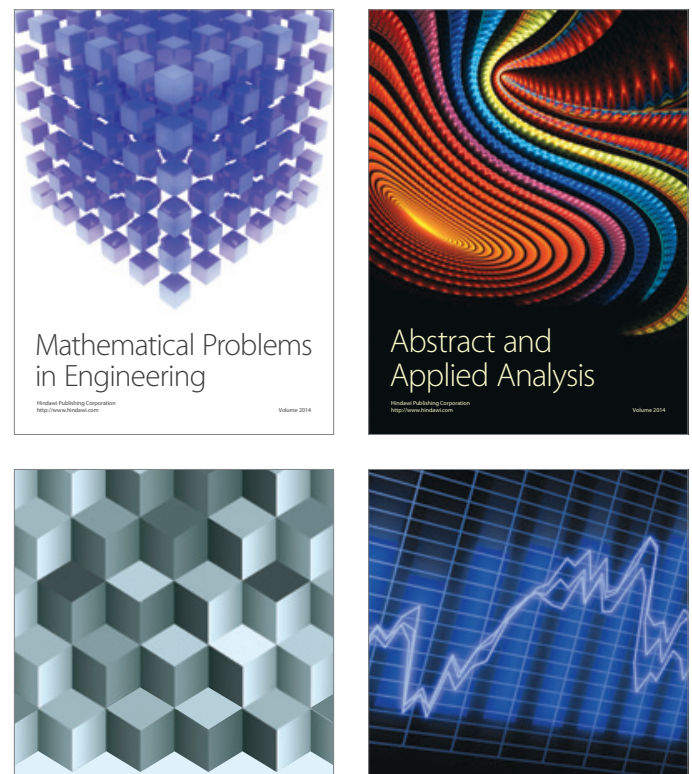

Journal of

Function Spaces

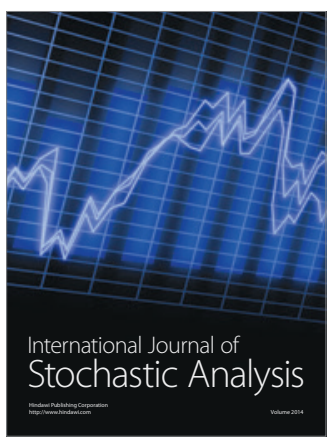

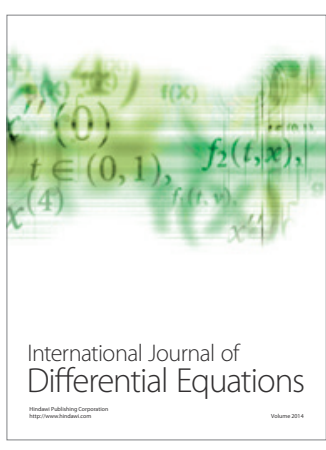
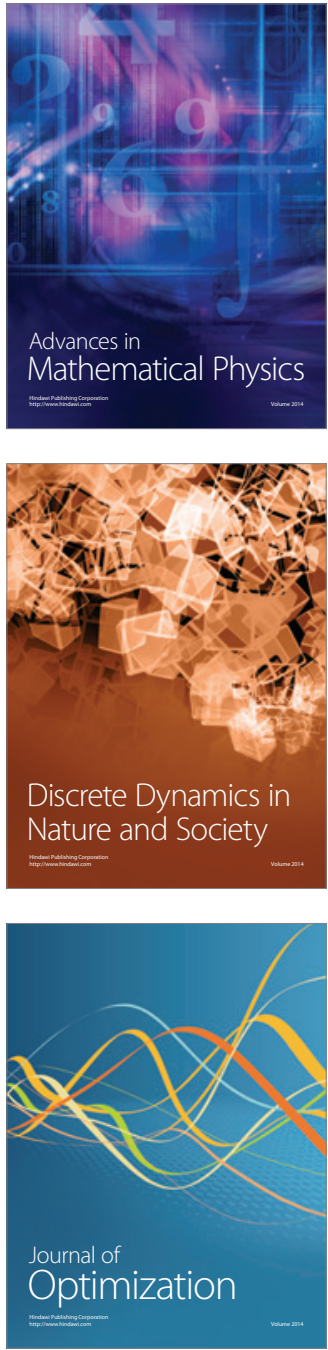